\title{
Contribution à la connaissance de la flore du Maroc septentrional : Maroc Atlantique nord, Rif, Littoral méditerranéen
}

\author{
Mathieu Chambouleyron \\ Emirates Center for Wildlife Propagation, Province de Boulemane, 33250 Missour, Maroc.
}

\section{Correspondencia}

M. Chambouleyron

e-mail: mchambouleyron@yahoo.fr

Recibido: 22 septiembre 2018

Aceptado: 22 septiembre 2019

Publicado on-line: 19 septiembre 2019

Editado por:T. Navarro

\section{Contribution to the knowledge of the flora of northern Morocco: Northern Atlantic Morocco, Rif, Mediterranean littoral}

Mots-clés: Chorologie, flore vasculaire, Maroc septentrional.

Key words: Chorology, vascular flora, northern Morocco.
Des prospections botaniques menées de 2010 à 2016 dans le Maroc septentrional (Fig. 1) ont permis la découverte de 29 taxons pour la plupart nouveaux localement.

Une partie des données présentées dans cet article se localisent aux marges de deux secteurs biogéographiques, rendant difficile le choix de l'attribution de nos observations à l'un ou à l'autre des secteurs concernés. C'est le cas :

De l'ouest de Ribat el Kheir, dans le bassin versant del'oued Sebou, secteurgéographiquement inclus dans les limites du Pré-Rif/Moyen Sebou (Man-1 sensu Fennane \& Ibn Tattou 2005), telles que cartographiées par Sauvage \& Vindt (19521954), Dobignard (2002) et Fennane \& Ibn Tattou (2005). Les données que nous présentons pour certains taxons sont en continuité géographique directe avec leur aire de distribution connue du

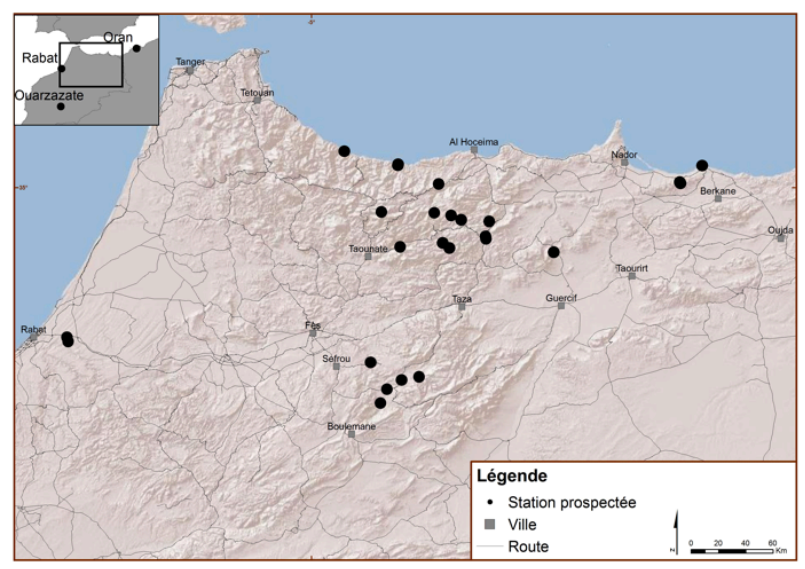

Figure 1. Localités prospectées. Surveyed localities.
Moyen-Atlas. Leur caractère nouveau pour le secteur du Moyen Sebou sera discuté au cas par cas, mais ils ne devront probablement y être admis que de manière très marginale ;

Des versants Est du Jbel Mezgout, à la limite entre le Rif oriental (R-3) et la basse-Moulouya $(\mathrm{Op}-1)$. Quelques taxons seront à rechercher plus à l'Ouest afin de confirmer leur présence dans le Rif oriental.

Les échantillons collectés sont conservés dans I'herbier CHAMB.

\section{ANGIOSPERMES DICOTYLÉDONES}

\section{Amaranthaceae}

\section{Atriplex semibaccata R.Br.}

Nouveau pour Man (ici Man-1) : Boulemane, Skoura (N33.58102, W4.54424), 890m, culture, 18.V.2016, «observation». Egalement observé à proximité, vers Tazouta par Alain Dobignard (http://www.floramaroccana. fr).

Confirmé pour le Rif (ici R-2), après les données fournies par Alain Dobignard à l'embouchure de l'oued Laou (http://www.floramaroccana.fr) : El Jebha, Massata, (N35.15422, W4.42880), 1m, plage, 05.V.2016, «observation». II a également été récemment signalé sur le littoral du Maroc nord-oriental par Dobignard (2009 : 14), $150 \mathrm{~km}$ plus à l'Est.

Taxon exotique, en expansion au Maroc (Fennane \& Ibn Tattou, 2005 : 142).

\section{Apiaceae}

Torilis nodosa subsp. webbii (Jury) Kerguélen

Nouveau pour R-2 : El Jebha, Amtar, (N35.23876, W4.78436), 5m, matorral, 05.V.2016, «observation». 


\section{Asteraceae}

Achillea santolinoides Lag. subsp. santolinoides

R-2 : El Jebha, Massata, (N35.14591, W4.43030), 20m, bord de piste, 05.V.2016, CHAMB s.n.

L'espèce (sensu lato) est déjà connue dans le Rif, mais la répartition des sous-espèces au Maroc est à préciser selon El Alaoui Faris \& Ibn Tattou (2014).

Anacyclus homogamos (Maire) Humphries

Nouveau pour le Rif (ici R-2 et R-3) : El Jebha, Amtar, (N35.23876, W4.78436), 1m, plage, 05.V.2016, CHAMB s.n. ; Targuist, Bni Hammart, (N34.83364, W4.18966), $1510 \mathrm{~m}$, matorral, 06.V.2016, CHAMB s.n. ; Targuist, Bni Hammart, (N34.81728, W4.07822), 1440m, matorral, 06.V.2016, «observation» ; Aknoul, Ajdir, (N34.78862, W4.01358), 1410m, matorral, 06.V.2016, «observation».

Confirmé dans LM-2, où il avait été indiqué par Kahouadji (1986 : 149) sur les bords de l'oued Moulouya entre Mechra Hommadi et Zaïo : Berkane, Ras el ma, (N35.14540, W2.42376), 30m, rocher, 11.V.2010, «observation».

Scorzonera caespitosa subsp. Iongifolia (Emb. \& Maire) Dobignard

Nouveau pour le Rif (ici R-2) : Issaguen (Jbel Tidighine), (N34.83974, W4.53839), 2020m, rocher, 21.VII.2010, CHAMB s.n. (det. M. Ibn Tattou). L'espèce s.l. est déjà connue du Rif, nous précisons donc ici uniquement la répartition de cette sous-espèce.

\section{Brassicaceae}

\section{Isatis tinctoria L.}

Nouveau pour le Rif (ici R-3) : Targuist, Bni Hammart, (N34.83364, W4.18966), 1510m, culture, 06.V.2016, «observation».

\section{Crassulaceae}

\section{Umbilicus patens Pomel}

Man-1 : Sefrou, El Menzel, (N33.84927, W4.61065), 730m, rocher, 12.V.2016, CHAMB s.n.

R-3 : Taïneste, (N34.60282, W4.09148), 990m, rocher, 02.VI.2013, CHAMB s.n.

Nos récoltes présentent des feuilles inférieures à pétiole inséré à proximité du centre du limbe, des pédoncules floraux courts (< calice), des fleurs étalées à légèrement nutantes, des corolles blanchâtres plutôt globuleuses $(3,5-5 \times 2,5-3,1 \mathrm{~mm})$ et des carpelles allongés. Si l'on suit le traitement proposé par Maire (1977: 273-277) et les taxons retenus par Dobignard \& Chatelain (2011), elles se rattachent à U. patens.

\section{Caryophyllaceae}

\section{Silene obtusifolia Willd.}

R-2 : El Jebha, Massata, (N35.15422, W4.42880), 1m, plage, 05.V.2016, CHAMB s.n. ; El Jebha, Amtar, (N35.23876, W4.78436), 1m, plage, 05.V.2016, «observation».
Taxon cité sur littoral rifain par Ibn Tattou (1999a), puis seulement de la péninsule tingitane par Fennane \& Ibn Tattou (2005 : 131).

\section{Cistaceae}

Helianthemum ledifolium subsp. apertum (Pomel) Greuter \& Burdet

Nouveau pour le Rif (ici R-3) : Aknoul, (N34.66363, W3.84879), 1270m, matorral, 05.V.2012, CHAMB s.n.

Bien que l'espèce sensu lato soit citée dans le Rif (Soriano, 2002 ; Fennane \& Ibn Tattou, 2005 : 155), cette sous-espèce n'y avait jusqu'alors pas été indiquée.

Cela dit, savaleur taxonomique, basée sur la longueur des pédoncules floraux et la densité de l'inflorescence (Raynaud, 1992 ; Raynaud, 1999), nous semble bien faible. L'autre sous-espèce (subsp. ledifolium) a été récoltée en mélange au sein de la même population le même jour (CHAMB s.n.) avec des intermédiaires entre les deux taxons.

\section{Fabaceae}

Astragalus reinii subsp. nemorosus (Batt.) Maire

Nouveau pour R-3 : Aknoul (Azrou akchar), (N34.77768, W3.82690), 1700m, éboulis, 01.VI.2013, CHAMB s.n. ; Targuist, Bni Hammart, (N34.81728, W4.07822), 1440m, bord de piste, 06.V.2016, CHAMB s.n.

Coronilla minima subsp. lotoides (W.D.J. Koch) Nyman Nouveau pour le Rif (ici R-3) : Aknoul (Azrou akchar), (N34.77768, W3.82690), 1700m, yeuseraie, 01.VI.2013, CHAMB s.n.

Notre récolte présente des gousses (pas tout à fait mûres) dépassant $3 \mathrm{~cm}$ de longueur, ce qui nous conduit à l'attribuer à cette sous-espèce plutôt qu'à la sousespèce minima.

\section{Melilotus albus Medik.}

Nouveau pour Man (ici Man-1) : Boulemane, Skoura, (N33.58102, W4.54424), 890m, culture, 18.V.2016, CHAMB s.n.

Au Maroc, ce taxon n'est cité que de la péninsule tingitane (Rif occidental) par Taleb (2002), donnée reprise par Fennane \& Ibn Tattou (2005: 215) et Ibn Tattou (2007). II a également été observé par Alain Dobignard (com. pers.) dans les régions de Chefchaouen et de Ouezzane.

Ulex parviflorus subsp. africanus (Webb) Greuter Nouveau pour R-3 : Targuist, Bni Hdifa, (N35.02400, W4.16062), 990m, matorral, 06.V.2016, CHAMB s.n.

\section{Lamiaceae}

Ballota hirsuta Benth. subsp. hirsuta

Nouveau pour Man (ici Man-1) : Sefrou, El Menzel, (N33.84927, W4.61065), 730m, matorral, 12.V.2016, CHAMB s.n.

La subsp. intermedia (Batt.) Patzak est retenue par 
Dobignard \& Chatelain (2012 : 264), mais ne l'est pas par Ouyahya (2007) qui l'inclue dans la subsp. maroccana (Murb.) Patzak et pour qui ces deux taxons auraient alors une tige à pubescence dominée par des poils étoilésfasciculés. Notre récolte présente une pubescence de la tige nettement dominée par des poils basifixes simples et longs, et 18-23 fleurs par verticillastre. Si l'on suit les valeurs données par Dobignard $(2009: 57)$ relatives à la densité florale des verticillastres, alors notre récolte se rapproche de la subsp. hirsuta.

\section{Marrubium alysson L.}

Nouveau pour Man (ici Man-1) : Boulemane, Skoura, (N33.58102, W4.54424), 890m, culture, 18.V.2016, «observation».

Thymus zygis subsp. gracilis (Boiss.) R. Morales Nouveau pour le Rif (ici R-3) : Taza, Taïneste, (N34.63623, W4.13523), 1100m, pinède, 13.V.2016, CHAMB s.n.

\section{Malvaceae}

\section{Malope trifida Cav.}

R-3 : Taza, Taïneste, (N34.63623, W4.13523), $1100 \mathrm{~m}$, culture, 13.V.2016, CHAMB s.n.

Taxon indiqué sur la totalité de la chaîne rifaine par Ibn Tattou (1999b), puis seulement dans la péninsule tingitane par Fennane \& Ibn Tattou (2005 : 289).

\section{Orobanchaceae}

\section{Orobanche gracilis Sm.}

Nouveau pour Man (ici Man-1) : Sefrou, El Menzel, (N33.84931, W 4.61060), 730m, matorral, 12.V.2016, CHAMB s.n. (det. G. Moreno Moral) ; Ribat el Kheir (N33.89545, W4.43844), 920m, yeuseraie, 12.V.2016, CHAMB s.n. (det. G. Moreno Moral).

\section{Plantaginaceae}

\section{Misopates cf oranense (Faure) D.A. Sutton}

Nouveau pour Man (ici Man-1) : Boulemane, Skoura, Adrej, (N33.67127, W4.50217), 830m, matorral, 11.V.2016, CHAMB s.n.

Notre récolte se rapporte à Antirrhinum orontium var. flavum Batt. \& Pit., avec une tige ramifiée atteignant $63 \mathrm{~cm}$ de haut, glabre sauf dans sa partie supérieure au niveau de l'inflorescence où elle présente une pilosité glanduleuse courte avec quelques poils simples longs, des feuilles oblongues-linéaires atteignant $5,5 \times 0,6 \mathrm{~cm}$, des corolles jaunes longues de $13-14 \mathrm{~mm}$, et une pilosité double de l'inflorescence allant jusqu'à $2,5 \mathrm{~mm}$ de long (Fig. 2).

Contrairement à l'avis de Maire (1936: 251), ce taxon est donné comme synomyme hétérotype de Antirrhinum orontium var. oranense Faure par le site internet des Conservatoire et Jardin Botaniques de la Ville de Genève et South African National Biodiversity Institute (2015), inclus tous deux sous Misopates oranense (Faure) D.A. Sutton.

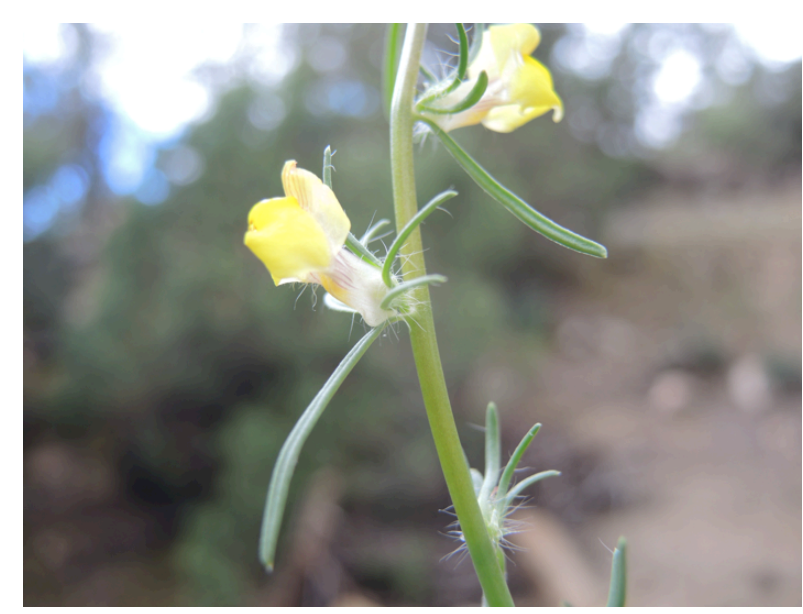

Figure 2. Misopates cf oranense, 11/05/2016, Adrej. Misopates cf oranense, 11/05/2016, Adrej.

L'aire de distribution principale de Misopates oranense se situerait de l'ouest algérien (Oranie) (Sutton, 1988 : 148) aux reliefs frontaliers marocains (Faure, 1941 : 197 ; Kahouadji, 1986 : 136). Cependant, Dobignard (2009: 75) signale la récolte aux environs de Sefrou (Moyen-Atlas), d'un taxon qu'il attribue à $M$. oranense, après l'avoir comparé avec les spécimens d'Algérie déposés à I'herbier MPU. Antirrhinum orontium var. flavum est cité du Moyen-Atlas par Maire (1936, I.c.) et doit sans doute correspondre au taxon cité par Dobignard (2009, I.c.), tout comme notre récolte.

Comme souligné par Dobignard (2009, I.c.), une étude plus poussée devrait être entreprise pour éclaircir cette question : sommes-nous en présence du même taxon qui serait alors distribué sur deux secteurs géographiques disjoints et relativement éloignés? Ou a-t-on affaire à deux taxons différents, comme le pensait Maire (1936, I.c.) ? La mise en culture de la plante médiatlasique et de celle de la frontière algéromarocaine serait utile afin d'avancer sur la question.

Notre récolte, localisée aux marges de Man, est en continuité géographique des stations médiatlasiques signalées par Dobignard (2009, I.C.) et Maire (1936, I.c).

\section{Plumbaginaceae}

\section{Limonium echioides (L.) Mill.}

Nouveau pour Man (ici Man-1) : Boulemane, Skoura, Adrej, (N33.67127, W4.50217), 830m, matorral, 11.V.2016, «observation».

Ce taxon est signalé non loin dans le Moyen-Atlas (environs de Skoura) par Fennane (1999) et Fennane \& Ibn Tattou (2005 : 315). Egalement observé par Alain Dobignard (com. pers.) entre Skoura et Tazouta.

\section{Primulaceae}

Lysimachia monelli (L.) U. Manns \& Anderb. subsp. monelli

Nouveau pour R-3 : Taza, Taïneste, (N34.63623, W4.13523), 1100m, culture, 13.V.2016, «observation». 


\section{Ranunculaceae}

\section{Ranunculus fibrosus Pomel}

Nouveau pour LM-2 : Zaïo, Tamdat Sidi Elbachir (Kebdana), (N35.02688, W2.56568), 740m, matorral, 20.IV.2016, CHAMB s.n.

L'étude et la distribution au Maroc des taxons du groupe $R$. paludosus s.l. est à revoir selon Dobignard (2009 : 10). Les trois spécimens de notre récolte présentent des racines nettement revêtues de restes fibreux, suffisamment enchevêtrés depuis les racines napiformes jusqu'au collet pour le distinguer $R$. paludosus Poir. s.s., d'après les données fournies par Dobignard (2013). Cela dit, la distinction de ce taxon avec $R$. flabellatus Desf. (récemment intégré à $R$. paludosus par Dobignard 2016 : 166), sur base de la densité du manchon fibreux reste peu évidente sur notre récolte où, bien qu'étant nettement développée, elle n'atteint pas la densité visible sur le scan du type de $R$. fibrosus présenté par Dobignard (2009: 15).

A proximité de là où nous l'avons récolté, ce taxon est cité par Dobignard (2009, I.c.) dans la région de Melilla (LM-1) et les monts de Debdou (Om-3).

\section{Ranunculus spicatus subsp. fontqueri Romo}

R-3 : Aknoul, Ajdir, (N34.78862, W4.01358), 1410m, rocher, 06.V.2016, CHAMB s.n.

Taxon indiqué dans le Rif par Ouyahya (1999), puis seulement sur le Jbel Tasnot (Rif occidental) et à rechercher ailleurs dans la chaîne rifaine par Fennane \& Ibn Tattou (2005 : 334).

\section{Resedaceae}

\section{Reseda alba L. subsp. alba}

R-3 : Saka (Jbel Mezgout), (N34.57538, W3.40296), 700m, pelouse, 17.III.2012, CHAMB s.n. ; Aknoul, (N34.67813, W3.85177), 1300m, matorral, 05.V.2012, CHAMB s.n.

Cette sous-espèce est citée dans le Rif par lbn Tattou (1999c), puis la répartition des sous-espèces de $R$. alba au Maroc est indiquée «à préciser » par Fennane \& Ibn Tattou (2005 : 335).

\section{Rubiaceae}

\section{Oldenlandia capensis $L$. f.}

Man-3 : Rabat, Souk Larbâa Essehoul (Maâmora), (N34.01446, W6.61247), 160m, daya, 12.VI.2010, CHAMB s.n.

Taxon uniquement connu de quelques dayas de la Maâmora, où il n'a plus été observé depuis les observations de Sauvage (1958), malgré un secteur considéré bien prospecté par les botanistes (Fennane 2018 : 30). Notre récolte semble provenir de la même daya que celle où Sauvage avait récolté ce taxon en 1956.

\section{Valerianaceae}

\section{Valeriana tuberosa $\mathrm{L}$.}

Nouveau pour LM (ici LM-2) : Zaïo, Tamdat Sidi
Elbachir (Kebdana), (N35.02688, W2.56568), 740m, matorral, 20.IV.2016, CHAMB s.n.

\section{ANGIOSPERMES MONOCOTYLÉDONES}

\section{Asparagaceae}

Anthericum baeticum (Boiss.) Boiss.

Nouveau pour LM-2 : Zaïo, Tamdat Sidi Elbachir (Kebdana), (N35.03727, W2.57044), 670m, rocher, 20.IV.2016, «observation».

Confirmation de la présence de ce taxon dans LM où il était à rechercher selon Ibn Tattou (2014).

\section{Hyacinthaceae}

\section{Ornithogalum sessiliflorum Desf.}

Nouveau pour Man (ici Man-1) : Sefrou, El Menzel, (N33.84927, W4.61065), 730m, matorral, 12.V.2016, «observation».

Ce taxon présente ici une extension de son aire aux marges de Man, depuis le Moyen-Atlas où il est mieux distribué

\section{Poaceae}

Gastridium phleoides (Nees \& Meyen) C.E. Hubb.

Nouveau pour Man-3 : Rabat, Souk Larbâa Essehoul (Maâmora), (N33.98791, W6.60564), 140m, oliveraie, 13.VI.2010, CHAMB s.n.

Ce taxon n'est pas reconnu au Maghreb par Dobignard \& Chatelain (2010: 298) et par le site internet des Conservatoire et Jardin Botaniques de la Ville de Genève et South African National Biodiversity Institute (2015). Sa présence en Méditerranée occidentale a été discutée par Romero García (1996), qui lui distingue deux sous-espèces : phleoides et lainzii Romero García, cette dernière par la suite ramenée au niveau spécifique, Gastridium lainzii (Romero García) Romero Zarco, par Romero Zarco (2013).

L'unique spécimen de notre récolte présente des lemmes à dos entièrement poilu, à peine prolongées par des aristules terminales très réduite ( $\leq 0,2 \mathrm{~mm}$ long), et à arête longue de 4,5-5,5 mm. En suivant les données de Romero García (1996), nous attribuons donc notre récolte à Gastridium phleoides (Nees \& Meyen) C.E. Hubb.

\section{Remerciements}

Nous adressons nos vifs remerciements à MM. Alain Dobignard qui a confirmé notre identification de Ranunculus fibrosus et a très aimablement accepté de relire le manuscrit de cet article en nous faisant part de ses précieux commentaires, Mohammed Ibn Tattou (Institut Scientifique de Rabat) qui a déterminé notre récolte de Scorzonera caespitosa subsp. longifolia, et Gonzalo Moreno Moral qui a déterminé nos Orobanches. Nous remercions également l'Emirates Center for Wildlife Propagation (ECWP) et I'International Fund for Houbara Conservation (IFHC) pour la mise à disposition de leurs 
ressources (matériel, fonds bibliographique et herbier).

\section{Références}

Conservatoiree Jardin Botaniques de la Ville de Genève \& South African National Biodiversity Institute (2015). Base de données des plantes d'Afrique [en ligne]. http://www.ville-ge.ch/musinfo/bd/cjb/africa/ [Accès le 11 février 2017].

Dobignard, A. (2002). Contributions à la connaissance de la flore du Maroc et de l'Afrique du Nord. Nouvelle série. 1. Journal de botanique 20, 5-43.

Dobignard, A. (2009). Contributions à la connaissance de la flore du Maroc et de l'Afrique du Nord. Nouvelle série 2. La flore du Nord-Maroc. Journal de botanique 46-47, 1-136.

Dobignard, A (2013). Ranunculus paludosus subsp. flabellatus (Desf.) Dobignard. In A. Dobignard \& C. Chatelain, Index synonymique de la flore d'Afrique du nord. Dicotyledoneae : Oleaceae à Zygophyllaceae, 5, 369. Conservatoire et Jardin botaniques de la Ville de Genève, ECWP. Genève.

Dobignard, A. (2016). Iter maroccanum 2014. Compte rendu de la 49 è Session extraordinaire de la Société botanique du Centre-Ouest dans le Grand Atlas marocain. Evaxiana 2, 107-252.

Dobignard, A. \& Chatelain, C. (2010). Index synonymique de la flore d'Afrique du nord. Pteridophyta, Gymnospermae, Monocotyledoneae, 1. Conservatoire et Jardin botaniques de la Ville de Genève, ECWP. Genève.

Dobignard, A. \& Chatelain, C. (2011). Index synonymique de la flore d'Afrique du nord. Dicotyledoneae : Balsaminaceae - Euphorbiaceae, 3. Conservatoire et Jardin botaniques de la Ville de Genève, ECWP. Genève.

Dobignard, A. \& Chatelain, C. (2012). Index synonymique de la flore d'Afrique du nord. Dicotyledoneae : Fabaceae - Nymphaeaceae, 4. Conservatoire et Jardin botaniques de la Ville de Genève, ECWP. Genève.

El Alaoui Faris, F.Z. \& Ibn Tattou, M. (2014). Achillea L. In M. Fennane et al. (eds), Flore Pratique du Maroc, 3, 231. Travaux de l'Institut Scientifique, Série Botanique, $n^{\circ} 40$, Rabat.

Faure, A. (1941). Notes sur mes herborisations au Maroc Oriental. Bulletin de la Société d'histoire naturelle d'Afrique du Nord 31, 180-205.

Fennane, M. (1999). Limonium Miller. In M. Fennane et al. (eds), Flore Pratique du Maroc, 1 : 278. Travaux de l'Institut Scientifique, Série Botanique, n³6, Rabat.

Fennane, M. (2018). Éléments pour un Livre rouge de la flore vasculaire du Maroc, 9. Dicotyledonae (Polygalaceae - Zygophyllaceae) (Version 1). Editions Tela-Botanica, Montpellier.

Fennane, M. \& Ibn Tattou, M. (2005). Flore vasculaire du Maroc : inventaire et chorologie, Pteridophyta, Gymnospermae, Angiospermae p.p., 1. Travaux de I'Institut Scientifique, Série Botanique, 37, Rabat.

Ibn Tattou, M. (1999a). Silene L. In M. Fennane et al. (eds), Flore Pratique du Maroc, 1 : 242. Travaux de I'Institut Scientifique, Série Botanique, 36, Rabat.

Ibn Tattou, M. (1999b). Malope L. In M. Fennane et al. (eds), Flore Pratique du Maroc, 1 : 294. Travaux de l'Institut Scientifique, Série Botanique, 36, Rabat.

Ibn Tattou, M. (1999c). Reseda L. In M. Fennane et al. (eds), Flore Pratique du Maroc, 1, 442. Travaux de l'Institut Scientifique, Série Botanique, 36, Rabat.

Ibn Tattou, M. (2007). Melilotus Miller. In M. Fennane et al. (eds), Flore Pratique du Maroc, 2, 153. Travaux de I'Institut Scientifique, Série Botanique, 38, Rabat.

Ibn Tattou, M. (2014). Anthericum L. In M. Fennane et al. (eds), Flore Pratique du Maroc, 3, 378. Travaux de I'Institut Scientifique, Série Botanique, 40, Rabat.

Kahouadji, A. (1986). Recherches floristiques sur le massif montagneux des Beni-Snassene (Maroc oriental). Thèse Université des Sciences et Techniques du Languedoc, Montpellier.

Maire, R. (1936). Contribution à l'étude le la Flore de l'Afrique du Nord. Fascicule 24 (suite et fin). Bulletin de la Société d'histoire naturelle d'Afrique du Nord 27(7), 241-270.

Ouyahya, A. (1999). Ranunculus L. In M. Fennane et al. (eds), Flore Pratique du Maroc, 1 : 94. Travaux de I'Institut Scientifique, Série Botanique, 38, Rabat.

Ouyahya, A. (2007). Ballota L. In M. Fennane et al. (eds), Flore Pratique du Maroc, $2: 460$. Travaux de l'Institut Scientifique, Série Botanique, 38, Rabat.

Raynaud, C. (1992). Éléments pour la flore pratique du Maroc. Fascicule 2. Cistaceae. Naturalia Monspeliensis, Série Botanique, 56, 171-220.

Raynaud, C. (1999). Helianthemum Miller. In M. Fennane et al. (eds), Flore Pratique du Maroc, $1: 314$. Travaux de I'Institut Scientifique, Série Botanique, 36, Rabat.

Romero Garcia, A.T. (1996). Un taxon nuevo del género Gastridium P. Beauv. (Gramineae) en Andalucía occidental (España). Anales del Jardín Botánico de Madrid 54, 399-406.

Romero-Zarco, C. (2013). Notas sobre gramíneas del N de Marruecos. Acta Botanica Malacitana 38, 224-227.

Sauvage, C. (1958). Au sujet de la présence d'Oldenlandia capensis L. fil. dans les environs de Rabat. Comptes rendus des séances mensuelles de la Société des Sciences Naturelles et Physiques du Maroc 7, 167-168.

Sauvage, C. \& Vindt, J. (1952-1954). Flore du Maroc analytique, descriptive et illustrée 1-2. Travaux de I'Institut Scientifique Chérifien. Éditions Internationales, Tanger.

Soriano, I. (2002). Helianthemum Mill. In B. Valdés et al. (eds), Catalogue des Plantes Vasculaires du Nord du Maroc, incluant des clés d'identification, 1, 209. CSIC, Madrid.

Taleb, A. (2002). Melilotus Mill. In B. Valdés et al. (eds), Catalogue des Plantes Vasculaires du Nord du Maroc, incluant des clés d'identification, 1, 356. CSIC, Madrid.

Valdés, B., Rejdali, M., Achhal El Kadmiri, A., Jury, J.L. \& Montserrat, J.M. (eds.) (2002). Catalogue des plantes vasculaires du Nord du Maroc, incluant des clés d'identification. CSIC, Madrid. 